Proceedings of the

International Geometry Center

Vol. 13, no. 1 (2020) pp. 1-8

\title{
Classification of curves on de Sitter plane
}

\author{
Irina Streltsova
}

\begin{abstract}
In 1917, de Sitter used the modified Einstein equation and proposed a model of the Universe without physical matter, but with a cosmological constant. De Sitter geometry, as well as Minkowski geometry, is maximally symmetrical. However, de Sitter geometry is better suited to describe gravitational fields. It is believed that the real Universe was described by the de Sitter model in the very early stages of expansion (inflationary model of the Universe).

This article is devoted to the problem of classification of regular curves on the de Sitter space. As a model of the de Sitter plane, the upper half-plane on which the metric is given is chosen. For this purpose, an algebra of differential invariants of curves with respect to the motions of the de Sitter plane is constructed. As it turned out, this algebra is generated by one second-order differential invariant (we call it by de Sitter curvature) and two invariant differentiations. Thus, when passing to the next jets, the dimension of the algebra of differential invariants increases by one. The concept of regular curves is introduced. Namely, a curve is called regular if the restriction of de Sitter curvature to it can be considered as parameterization of the curve. A theorem on the equivalence of regular curves with respect to the motions of the de Sitter plane is proved. The singular orbits of the group of proper motions are described.
\end{abstract}

Анотація. В 1917 році де Сіттер використав модифіковане рівняння Ейнштейна і запропонував модель Всесвіту без фізичної матерії але 3 космологічною постійною. Геометрія Де Сіттера, як і геометрія Мінковського, є максимально симетричною. Однак геометрія де Сіттера краще підходить для опису гравітаційних полів. Зокрема, вважається, що розширення Всесвіту на самих ранніх стадіях може бути описане саме моделлю де Сіттера (інфляційна модель Всесвіту).

Дана стаття присвячена проблемі класифікації регулярних кривих в просторі де Сіттера, а в якості моделі площини де Сіттера обрана верхня півплощина, на якій задана метрика. Для розв'язання поставленої задачі побудовано алгебру диференціальних інваріантів кривих відносно рухів

$U D C 514.75+517.95$

Keywords: differential invariants, invariant differentiations, de Sitter plane, jets. DOI: http://dx.doi.org/10.15673/tmgc.v13i1.1683 
площини де Сіттера. Як виявилось, ця алгебра породжена одним диференціальним інваріантом другого порядку (ми називаємо його кривиною де Cimmepa) та двома інваріантними диференціюваннями. Таким чином, при переході до наступних струменів розмірність алгебри диференціальних інваріантів збільшується на одиницю.

Також в роботі введено поняття регулярних кривих. А саме, криву на площині де Сіттера називають регулярною, якщо обмеження кривини де Сіттера на неї можна розглядати як параметризацію кривої. Доведено теорему про еквівалентність регулярних кривих відносно рухів площини де Сіттера та описано сингулярні орбіти групи власних рухів.

\section{INTRODUCTION}

Like Minkowski geometry, de Sitter geometry is one of the formalizations of the theory of relativity. Model of de Sitter universe was proposed in $[3,2]$. Unlike Minkowski geometry, it is better suited for descriptions of a nontrivial gravitational field, in particular, the expansion effect of the universe [7].

At the same time, there is much in common between Minkowski and de Sitter geometries: both of them are maximally symmetrical. The group of motions of Minkowski space, that is, the group of transformations preserving the metric, is the 10-parameters Poincaré group, consisting of four translations (three spatial and one temporal) three purely spatial rotations and three space-time rotations. The last six transformations form a subgroup of the Poincaré group (the group of Lorentz transformations).

De Sitter's space also has a 10-parametric dimensional group of motions. A review of de Sitter geometry is given in [6].

This article is devoted to the problem of classification of curves in 2dimensional de Sitter space. We consider the upper half-plane

$$
M=\mathbb{R}_{+}^{2}=\left\{(x, y) \in \mathbb{R}^{2} \mid y>0\right\},
$$

with the metric

$$
g_{\mathrm{S}}=\frac{d x^{2}-d y^{2}}{y^{2}} .
$$

as a model of this space. This half-plane will be called de Sitter plane.

The proper motions generate a 3-dimensional Lie group, which we denote by $G_{\mathrm{S}}$ and call de Sitter group. This group is generated by translations by $x$, the transformation

$$
\Phi_{t}: x \longmapsto-\frac{2\left(-t y^{2}+t x^{2}-2 x\right)}{t^{2} x^{2}-4 t x-t^{2} y^{2}+4}, \quad y \longmapsto \frac{4 y}{t^{2} x^{2}-4 t x-t^{2} y^{2}+4}
$$

and homotheties. 
The corresponding Lie algebra $\mathcal{G}_{\mathrm{S}}$ is generated by the vector fields

$$
X=\frac{\partial}{\partial x}, \quad Y=\frac{1}{2}\left(x^{2}+y^{2}\right) \frac{\partial}{\partial x}+x y \frac{\partial}{\partial y}, \quad H=x \frac{\partial}{\partial x}+y \frac{\partial}{\partial y} .
$$

Consider the problem of classifying curves on de Sitter plane that defined as function graphs $y=f(x)$.

\section{The Dimension of the Algebra of Differential invariants}

Let $J^{k}(\pi)$ be the space of $k$-jets of curves bundle, and let $x, y_{0}, y_{1}, \ldots, y_{k}$ be the local canonical coordinates in the space $J^{k}(\pi)$.

The generating functions [4] of the vector fields $X, Y, H$ are

$$
h_{X}=-y_{1}, \quad h_{Y}=x y_{0}-\frac{y_{1}}{2}\left(x^{2}+y_{0}^{2}\right), \quad h_{H}=y_{0}-x y_{1} .
$$

respectively.

A function $J$ on the space of $k$-jets is called a differential invariant of order $\leqslant k$ of the Lie group $G_{\mathrm{S}}$, if $\varphi^{*}(J)=J$ for each transformation $\varphi \in G_{\mathrm{S}}$, [1].

Define the projection from $J^{k}(\pi)$ to $J^{0}(\pi)$ by:

$$
\pi_{k}:\left(x, y_{0}, y_{1}, \ldots, y_{k}\right) \longmapsto\left(x, y_{0}\right) .
$$

Let $a$ be some point on the manifold $M$. Since the Lie group $G_{\mathrm{S}}$ acts transitively on this manifold, $N_{a}^{k}=\pi_{k}^{-1}(a)$ is a smooth manifold with coordinates $y_{1}, \ldots, y_{k}$. Let $G_{a}=\left\{g \in G_{\mathrm{S}} \mid g a=a\right\} \subset G_{\mathrm{S}}$ be the isotropy group of the point $a$. Then transformations from the prolonged Lie group $G_{a}^{(k)}$ preserve the manifold $N_{a}^{k}$.

Let $\mathcal{G}_{a} \subset \mathcal{G}_{\mathrm{S}}$ be the corresponding to $G_{a}$ Lie subalgebra. We call this subalgebra the stabilizer of the point $a$. Note that the point $a$ is a singular point for each vector field from $\mathcal{G}_{a}$.

Let us describe the stabilizer $\mathcal{G}_{a}$ of the point $a\left(a_{x}, a_{y}\right) \in M$. For this purpose take a vector field $Z_{a} \in \mathcal{G}_{a}$ with singularity at the point $a$, so

$$
Z_{a}=\alpha X+\beta Y+\gamma H
$$

for some constants $\alpha, \beta, \gamma$, i.e.

$$
Z_{a}=\left(\gamma x+\frac{\beta}{2}\left(x^{2}+y_{0}^{2}\right)+\alpha\right) \frac{\partial}{\partial x}+\left(\gamma y_{0}+\beta x y_{0}\right) \frac{\partial}{\partial y_{0}} .
$$

Since the point $a$ is singular, we have

$$
\gamma a_{x}+\frac{\beta}{2}\left(a_{x}^{2}+a_{y}^{2}\right)+\alpha=0, \quad \gamma a_{y}+\beta a_{x} a_{y}=0 .
$$

Therefore

$$
\alpha=\beta \frac{a_{x}^{2}-a_{y}^{2}}{2}, \quad \gamma=-\beta a_{x},
$$


where $\beta$ is arbitrary parameter. Put $\beta=1$. Then we get

$$
Z_{a}=\frac{a_{x}^{2}-a_{y}^{2}}{2} X+Y-a_{x} H .
$$

The generating function of the vector field $Z_{a}$ is

$$
h_{Z_{a}}=\frac{1}{2}\left(-y_{0}^{2}-\left(x-a_{x}-a_{y}\right)\left(x-a_{x}+a_{y}\right)\right) y_{1}+\left(x-a_{x}\right) y_{0} .
$$

So, $\operatorname{dim} \mathcal{G}_{a}=1$ and $\mathcal{G}_{a}=\mathbb{R} Z_{a}$.

Let $S_{Z_{a}}$ be the evolutionary part of the vector fields $Z$. Recall, [4], that

$$
S_{Z_{a}}=h_{Z} \frac{\partial}{\partial y_{0}}+\frac{d h_{Z}}{d x} \frac{\partial}{\partial y_{0}}+\frac{d^{2} h_{Z}}{d x^{2}} \frac{\partial}{\partial y_{0}}+\cdots+\frac{d^{k} h_{Z}}{d x^{l}} \frac{\partial}{\partial y_{k}},
$$

where

$$
\frac{d}{d x}=\frac{\partial}{\partial x}+y_{1} \frac{\partial}{\partial y_{0}}+y_{2} \frac{\partial}{\partial y_{1}}+\cdots
$$

is the operator of total differentiation and $\frac{d^{j}}{d x^{j}}$ is the $j$-th power of $\frac{d}{d x}$.

Notice that the vector fields $S_{Z_{a}}^{(k)}$ is tangent to the submanifold

$$
N_{a}^{k} \subset J^{k}(\pi)
$$

Let $s_{f}=\left\{x, y_{0}=f(x)\right\}$ be a curve on $M$. Its prolongation

$$
s_{f}^{(k)}=\left\{x, y_{0}=f(x), y_{1}=f^{\prime}(x), \ldots, y_{k}=f^{(k)}(x)\right\}
$$

intersects $N_{a}^{k}$ at a unique point. Therefore, the curve $s_{f}^{(k)}$ is uniquely determined by the point of its intersection with $N_{a}^{k}$. Let $\theta=\left[s_{f}\right]_{a}^{k}$ be this point.

A dimension of the orbit of the curve $s_{f}^{(k)}$ at the point $\pi_{k}^{-1}(a)$ is the dimension of the orbit of the point $\theta$ on the submanifold $N_{a}^{k}$.

The dimension of the $G_{a}^{(k)}$-orbit of the point $\theta$ is the rank of the $1 \times(k+1)$ matrix

$$
\left(0, a_{y}\left(1-y_{1}^{2}\right),\left(1-y_{1}^{2}-3 a_{y} y_{2}\right) y_{1}\right)
$$

Since $a_{y}>0$, the rank is zero if $1-y_{1}^{2}=y_{2}=0$. Otherwise it equals 1 . Therefore, the set

$$
\Sigma_{\mathrm{S}}=\left\{1-y_{1}^{2}=0, y_{2}=0\right\}
$$

consists of singular points of the $G_{a}^{(k)}$-orbit.

The general curve has no singular points, therefore the codimension of its orbit is equal to 1.

Theorem 2.1. The dimension of the algebra of differential invariants of order $\leqslant k$ with respect to the Lie group $G_{\mathrm{S}}$ is $k-1$. 


\section{Curvatures of Curves on de Sitter plane}

According to Theorem 2.1, the first differential invariants arise in the second order. Let us find them. The prolongations of the vector fields $X, Y, H$ into $J^{2}(\mathbb{R})$ are

$$
\begin{aligned}
X^{(2)}= & \frac{\partial}{\partial x}, \\
Y^{(2)}= & \frac{1}{2}\left(x^{2}+y_{0}^{2}\right) \frac{\partial}{\partial x}+x y_{0} \frac{\partial}{\partial y_{0}}+\left(y_{0}-y_{0} y_{1}^{2}\right) \frac{\partial}{\partial y_{1}}+ \\
& +\left(y_{1}-y_{1}^{3}-3 y_{0} y_{1} y_{2}-x y_{2}\right) \frac{\partial}{\partial y_{2}}, \\
H^{(2)}= & x \frac{\partial}{\partial x}+y_{0} \frac{\partial}{\partial y_{0}}-y_{2} \frac{\partial}{\partial y_{2}} .
\end{aligned}
$$

Solving the system of differential equations

$$
X^{(2)}(J)=0, \quad Y^{(2)}(J)=0, \quad H^{(2)}(J)=0,
$$

we will find a first differential invariant

$$
J_{2}=\frac{y_{2} y_{0}+y_{1}^{2}-1}{\left|y_{1}^{2}-1\right|^{\frac{3}{2}}} .
$$

The function

$$
J_{2}(f)=\frac{f^{\prime \prime}(x) f(x)+\left(f^{\prime}(x)\right)^{2}-1}{\left|\left(f^{\prime}(x)\right)^{2}-1\right|^{\frac{3}{2}}} .
$$

is called a de Sitter curvature of the curve $y=f(x)$.

\section{Algebra of Differential invariants}

It is not hard to check that the operator

$$
\nabla_{\mathrm{S}}=\frac{y_{0}}{\sqrt{\left|y_{1}^{2}-1\right|}} \frac{d}{d x}
$$

is an invariant differentiation.

The third order invariant can be obtained by applying the operator $\nabla_{\mathrm{S}}$ to the invariant $J_{2}$ :

$$
J_{3}=\frac{y_{0}^{2}\left(y_{3} y_{1}^{2}-3 y_{2}^{2} y_{1}-y_{3}\right)}{\left(y_{1}^{2}-1\right)^{3}} .
$$

Using the invariant derivation $\nabla_{\mathrm{S}}$, we can construct higher order differential invariants:

$$
J_{4}=\nabla\left(J_{3}\right), \ldots, J_{k}=\nabla\left(J_{k-1}\right), \ldots
$$


Let us indicate the type of fourth order differential invariant in coordinates:

$$
\begin{gathered}
J_{4}=\frac{y_{0}^{2}}{\left(-1+y_{1}^{2}\right)^{\frac{9}{2}}}\left(2 y_{3} y_{1}^{5}+y_{0} y_{4} y_{1}^{4}-6 y_{2}^{2} y_{1}^{4}-4 y_{3} y_{1}^{3}-10 y_{0} y_{2} y_{3} y_{1}^{3}-\right. \\
-2 y_{0} y_{4} y_{1}^{2}+15 y_{0} y_{1}^{2} y_{2}^{3}+6 y_{1}^{2} y_{2}^{2}+10 y_{0} y_{1} y_{2} y_{3}+ \\
\left.+2 y_{1} y_{3}+y_{0} y_{4}+3 y_{0} y_{2}^{3}\right) .
\end{gathered}
$$

Theorem 4.1. The functions $J_{2}, J_{3}, \ldots, J_{k}, \ldots$ form the basis of local differential invariants of a curve with respect to the de Sitter group $G_{\mathrm{S}}$.

\section{Equivalence of CuRVES}

A curve $s$ on de Sitter is called regular if $\left|d J_{2}\right|_{s} \neq 0$. This means that the restriction $\left.J_{2}\right|_{s}$ of $J_{2}$ to $s$ can be taken as a new parameter on the curve. Therefore the restriction of the differential invariant $J_{3}$ to the curve $s$ can be represented as a function of $\tau$ :

$$
\left.J_{3}\right|_{s}=F(\tau) .
$$

Theorem 5.1. Suppose that the following conditions are satisfied for two regular curves $s$ and $\tilde{s}$ on the de Sitter plane:

(1) the functions $F(\tau)$ and $\tilde{F}(\tilde{\tau})$ do not vanish;

(2) the prolongations of the curves $s$ and $\tilde{s}$ to the space of 2-jets belong to the same connected component.

Then the curves s and $\tilde{s}$ are locally equivalent with respect to the movements of the de Sitter plane if and only if $F \equiv \tilde{F}$.

Proof. Let us prove necessity. Suppose that the curves $s$ and $\tilde{s}$ are locally equivalent. This means that there exists a transformation $\Phi \in G_{\mathrm{S}}$ that transforms the curve $s$ into $\tilde{s}$. Therefore the prolongations $s^{(2)}$ and $\tilde{s}^{(2)}$ of these curves to the 2 -jet space $J^{2}(\mathbb{R})$ lie on the same connected component. Moreover, since $J_{2}$ and $J_{3}$ are differential invariants, we have $F \equiv \tilde{F}$.

Prove that condition $F \equiv \tilde{F}$ is sufficient for local equivalence of the curves $s$ and $\tilde{s}$. Suppose that the curves $s$ and $\tilde{s}$ are graphs of the functions $y=f(x)$ and $y=\tilde{f}(x)$ respectively. The ordinary third order differential equation

$$
J_{3}=F\left(J_{2}\right),
$$

defines the hypersurface $E$ in the space $J^{3}(\mathbb{R})$. The functions $y=f(x)$ and $y=\tilde{f}(x)$ are solutions of this equation and the curves $s^{(3)}$ and $\tilde{s}^{(3)}$ lie on this hypersurface. Due to condition (2) of the theorem, the restrictions 
of the function $1-y_{1}^{2}$ to these curves are everywhere not zero. Therefore equation (5.1) can be solved with respect to the highest derivative:

$$
y_{3}=\frac{3 y_{0}^{2} y_{1} y_{2}^{2}+F\left(\frac{y_{2} y_{0}+y_{1}^{2}-1}{\left|y_{1}^{2}-1\right|^{\frac{3}{2}}}\right)\left(-1+y_{1}^{2}\right)^{3}}{y_{0}^{2}\left(-1+y_{1}^{2}\right)}
$$

The generating functions [4] of the vector fields $X, Y, H$ are

$$
h_{X}=-y_{1}, \quad h_{Y}=x y_{0}-\frac{x^{2} y_{1}+y_{1} y_{0}^{2}}{2}, \quad h_{H}=y_{0}-x y_{1}
$$

respectively. Therefore corresponding evolutionary vector fields are

$$
\begin{aligned}
S_{X}= & -y_{1} \frac{\partial}{\partial y_{0}}-y_{2} \frac{\partial}{\partial y_{1}}-y_{3} \frac{\partial}{\partial y_{2}} \\
S_{Y}= & \left(x y_{0}-\frac{x^{2} y_{1}+y_{1} y_{0}^{2}}{2}\right) \frac{\partial}{\partial y_{0}}+\left(y_{0}-y_{0} y_{1}^{2}-\frac{x^{2} y_{2}+y_{0}^{2} y_{2}}{2}\right) \frac{\partial}{\partial y_{1}}+ \\
& +\left(y_{1}-x y_{2}-y_{1}^{3}-3 y_{0} y_{1} y_{2}-\frac{x^{2} y_{3}+y_{0}^{2} y_{3}}{2}\right) \frac{\partial}{\partial y_{2}}, \\
S_{H}= & \left(y_{0}-x y_{1}\right) \frac{\partial}{\partial y_{0}}-x y_{2} \frac{\partial}{\partial y_{1}}-\left(y_{2}+x y_{3}\right) \frac{\partial}{\partial y_{2}} .
\end{aligned}
$$

The restrictions $\overline{S_{X}}, \overline{S_{Y}}, \overline{S_{H}}$ to the hypersurface $E$ are shuffling symmetries of equation (5.2). These restrictions are linearly independent if $\left(y_{1}^{2}-1\right) F \neq 0$. Due to condition (1) of the theorem we have $F(\tau) \neq 0$ and due to condition (2) we also have that $y_{1}^{2}-1 \neq 0$. Therefore the last inequality is true and the vector fields $\overline{S_{X}}, \overline{S_{Y}}, \overline{S_{H}}$ are linearly independent at each point of a connected component. So, the prolonged Lie group $G_{\mathrm{S}}$ acts transitively on each connected component.

Choose on each curve the points: $a_{0} \in s^{(3)}$ and $b_{0} \in \tilde{s}^{(3)}$. Since the shift by variable $x$ belongs to $G_{\mathrm{S}}$, we can assume that $x\left(a_{0}\right)=x\left(b_{0}\right)=a$. Thus, using a suitable transformation of this Lie group, we can achieve that $a_{0}=b_{0}$. So, without loss of generality, we can assume that the functions $y=f(x)$ and $y=\tilde{f}(x)$ being solutions of differential equation (5.2), have the same initial data. By the uniqueness theorem for solutions of differential equations, the functions $f$ and $\tilde{f}$ coincide at least locally.

Thus, using a suitable motion, the curve $s$ can be locally translated into the curve $\tilde{s}$.

Remark 5.2. The described method for finding differential invariants can be applied to problems of classifying curves in other geometries. In addition, it is applicable to the classification problems of certain classes of differential equations, see [5]. 


\section{REFERENCES}

[1] D. V. Alekseevskij, A. M. Vinogradov, V. V. Lychagin. Basic ideas and concepts of differential geometry, volume 28 of Encyclopaedia Math. Sci. Springer, Berlin, 1991.

[2] W. de Sitter. On the curvature of space. In Proceedings of the Royal Netherlands Academy of Arts and Science, volume 20, page 229, 1917.

[3] W. de Sitter. On the relativity of inertia: Remark concerning Einstein's latest hypothesis. In Proceedings of the Royal Netherlands Academy of Arts and Science, volume 19, pages $1217-1225,1917$.

[4] Alexei Kushner, Valentin Lychagin, Vladimir Rubtsov. Contact geometry and non-linear differential equations, volume 101 of Encyclopedia of Mathematics and its Applications. Cambridge University Press, Cambridge, 2007.

[5] Elena N. Kushner, Alexei G. Kushner, Alexey V. Samohin. Differential invariants of third order evolutionary non-linear PDEs. In 2019 Twelfth International Conference "Management of large-scale system development" (MLSD). IEEE, October 2019, doi: $10.1109 / \mathrm{mlsd} .2019 .8911039$.

[6] Ugo Moschella. The de Sitter and anti-de Sitter sightseeing tour. 47:120-133, 2006, doi: $10.1007 / 3-7643-7436-5$-4.

[7] Norbert Straumann. On the Cosmological Constant Problems and the Astronomical Evidence for a Homogeneous Energy Density with Negative Pressure, pages 7-51. Birkhäuser Basel, 2003, doi: 10.1007/978-3-0348-8075-6_2.

Received: December 12, 2019, accepted: February 18, 2020.

Irina Streltsova

Astrakhan State University, Astrakhan, 414056, Russia

Email: strelzova_i@mail.ru 